\title{
Degradación del suelo y desarrollo económico en la agricultura familiar de la parroquia Emilio María Terán, Píllaro
}

\section{Land degradation and economic development in family agriculture of the parish Emilio María Terán, Píllaro}

\author{
Christian Iván Montatixe Sánchez ${ }^{1 *}$, Mauricio David Eche Enriquez² \\ ${ }^{1}$ Asesor Agrícola Independiente, Machachi, Pichincha, Ecuador. \\ 凶 cmontatixeregion3@gmail.com. \\ https://orcid.org/0000-0001-7128-9049 \\ ${ }^{2}$ Universidad Regional Amazónica IKIAM, Vicerrectorado Académico, Carrera de Agroecología, \\ Vía Muyuna KM 7, Tena, Napo. \\ 凶mauricio.eche@ikiam.edu.ec. \\ https://orcid.org/0000-0002-7378-0066
}

\begin{abstract}
Resumen
Esta investigación tiene el propósito de determinar el efecto de la degradación del suelo sobre el ingreso económico de la agricultura familiar en la parroquia Emilio María Terán, cantón Píllaro, provincia de Tungurahua. Se realizó una investigación de campo, mediante la aplicación de encuestas cerradas que permitieron recolectar información referente a datos socioeconómicos, de producción y degradación del suelo. Éstas se aplicaron a 196 agricultores, jefes de hogar, de once barrios que conforman la parroquia. Es claro que el porcentaje de áreas degradadas que no cultivan corresponde al 70,9 \%; cualitativamente se demostró que existe una precaria situación económica, así como la presencia de unidades de producción agrícola (UPA) con áreas degradadas; sin embargo, los análisis cuantitativos determinan que la degradación no tiene efecto sobre el ingreso económico.
\end{abstract}

Palabras clave: suelo, erosión, antropogénica, productividad, ingresos.

\begin{abstract}
This research was aimed to quantify the effect of soil degradation on the economic income of family farming in the county of Emilio María Terán, Pillaro municipality in the Tungurahua province. A field survey was carried out using a closed-ended questionary that enabled to gather information about soil degradation, socio-economic and farm production. The survey was applied to 196 households' farmers heads from 11 neibourghoods. The percentage of the degraded soil that is not cultivated was $70.9 \%$. The qualitative analysis showed that farmers' economy was precarious together with degraded soils farms. However, from the quantitative analysis soil degradation was not associated with the farm economic income.
\end{abstract}

Keywords: ground, erosion, anthropogenetic, productivity, income. 


\section{Introducción}

La presente investigación se enfoca en el análisis de la degradación del suelo y el desarrollo económico de la agricultura familiar. La degradación del suelo se puede definir como el proceso de deterioro de las propiedades físicas, químicas y biológicas de la tierra, debido a la existencia de dos sistemas involucrados: el ecosistema natural y el medio social humano (Piscitelli, 2015).

Para analizar esta problemática es necesario mencionar sus causas. El ecosistema natural, por medio de los diferentes eventos climáticos extremos y persistentes, provoca la degradación del suelo. Así también, el entorno humano produce este efecto a través de la deforestación, eliminación de la cubierta vegetal, expansión urbana que da lugar a modificaciones en el uso del suelo y, como consecuencia, provoca cambios en su cobertura. Incluso la actividad agrícola puede tornarse insostenible debido a malas prácticas culturales, entre las que se encuentran: el uso y abuso de fertilizantes, pesticidas y equipos agrícolas, el excesivo pastoreo de animales, la inadecuada rotación de cultivos, mala aplicación de los sistemas de riego, que pueden considerarse como los principales procesos ejecutados por el hombre que ocasionan degradación del suelo (Espinoza Ramírez et al., 2011).

La investigación de esta problemática se realizó por el interés profesional de analizar una degradación avanzada del suelo, es decir, donde éste ha disminuido en $50 \%$ su capacidad para mantener una buena productividad; adicionalmente, con el paso del tiempo, el suelo presenta deterioro en sus propiedades físicas, químicas y biológicas, factores que determinan los bajos rendimientos de los cultivos; estos parámetros condicionan la situación socioeconómica de los habitantes que dependen de la actividad agrícola (Mateo Fernández, 2017). Así tambien, aporta información relevante sobre la degradación del suelo y el efecto que tiene sobre el desarrollo económico en la agricultura familiar. Dentro de los procesos de degradación del suelo, el tipo de erosión que con mayor frecuencia se produce en Ecuador es la erosión hídrica, causada por la exposición de los suelos a efectos climatológicos.

La degradación de los suelos entre los años 1982-2003 fue del 14,2\% del total de la tierra arable del territorio nacional, lo que representa $34.686 \mathrm{~km}^{2}$ (Morales, 2010). Según Jimenez (2011), cerca de 37,5 mil km², que representan el $15 \%$ de los sue- los, se encuentran en estado de erosión, es decir, la degradación del suelo ha aumentado en un $0,8 \%$, de los cuales en la región Sierra se aprecia el 25,9 $\%$; el $30 \%$ se presentó en la Costa, y el $44 \%$ en la Amazonia. En el sitio de estudio, la parroquia Emilio María Terán, ubicada en la provincia de Tungurahua, se encuentra afectado por la erosión el 28,98 \% del suelo arable, los lugares donde predomina este inconveniente son el bosque montano alto y la zona de Cutzatagua; con el 26,20\% de la superficie parroquial se encuentra afectada la quebrada Pucahuayco y la zona baja del barrio Quillán Alemán; los sectores de Juanillo y Quillán la Merced, constituyen el $32,6 \%$ del territorio que registra moderada tendencia a la erosión y, finalmente, el 12,22 \% tiene una baja tendencia a la erosión (GAD de Emilio María Terán, 2015).

Este proyecto de investigación se justifica y se basa en la percepción obtenida de los agricultores, ya que fue una investigación de tipo descriptivo, que se considera concluyente, dada su naturaleza cuantitativa, es decir, se planteó previamente con base en una encuesta cerrada que permitió recopilar información, la cual pudo atribuirse estadísticamente a una población, la misma que ayudo a definir con mayor precisión la opinión, la actitud y el comportamiento de los agricultores jefes de hogar a los que fue dirigida la encuesta sobre el tema en estudio.

\section{Materiales y métodos}

\subsection{Descripción del sitio de investigación}

La investigación se realizó en la parroquia Emilio María Terán, perteneciente al cantón Santiago de Píllaro, provincia de Tungurahua, ubicada a $1^{\circ} 12^{\prime} 15^{\prime \prime}$ - $1^{\circ} 14^{\prime} 28^{\prime \prime}$ de latitud norte, y $78^{\circ} 32^{\prime} 20^{\prime \prime}$ - 78'29'15" de longitud occidental. Sus límites territoriales son, al norte: San Miguelito, al sur: Chiquicha (cantón Pelileo) y los Andes (cantón Patate), al este: Baquerizo Moreno y, al oeste: San Miguelito e Izamba (cantón Ambato) (Figura 1) (GAD de Emilio María Terán, 2015). La zona presenta las siguientes condiciones climáticas: debido a su altitud, que va desde 2.481 a $2.726 \mathrm{~m}$ s.n.m., la temperatura promedio anual de la parroquia Emilio María Terán oscila entre los 14 y $15^{\circ} \mathrm{C}$, y posee una precipitación anual promedio de 1.000 a $2.000 \mathrm{~mm}$.

La población de la parroquia Emilio María Terán está conformada por 11 barrios, con 397 fa- 
milias, aproximadamente; el 48,74\% son hombres (733) y el 51,26\% (771) son mujeres, con un total de 1.504 habitantes. La distribución de la población por edad -hombres y mujeres-, se ubica entre los 20 y 64 años, señalándose que ésta es la población económicamente activa de la parroquia.

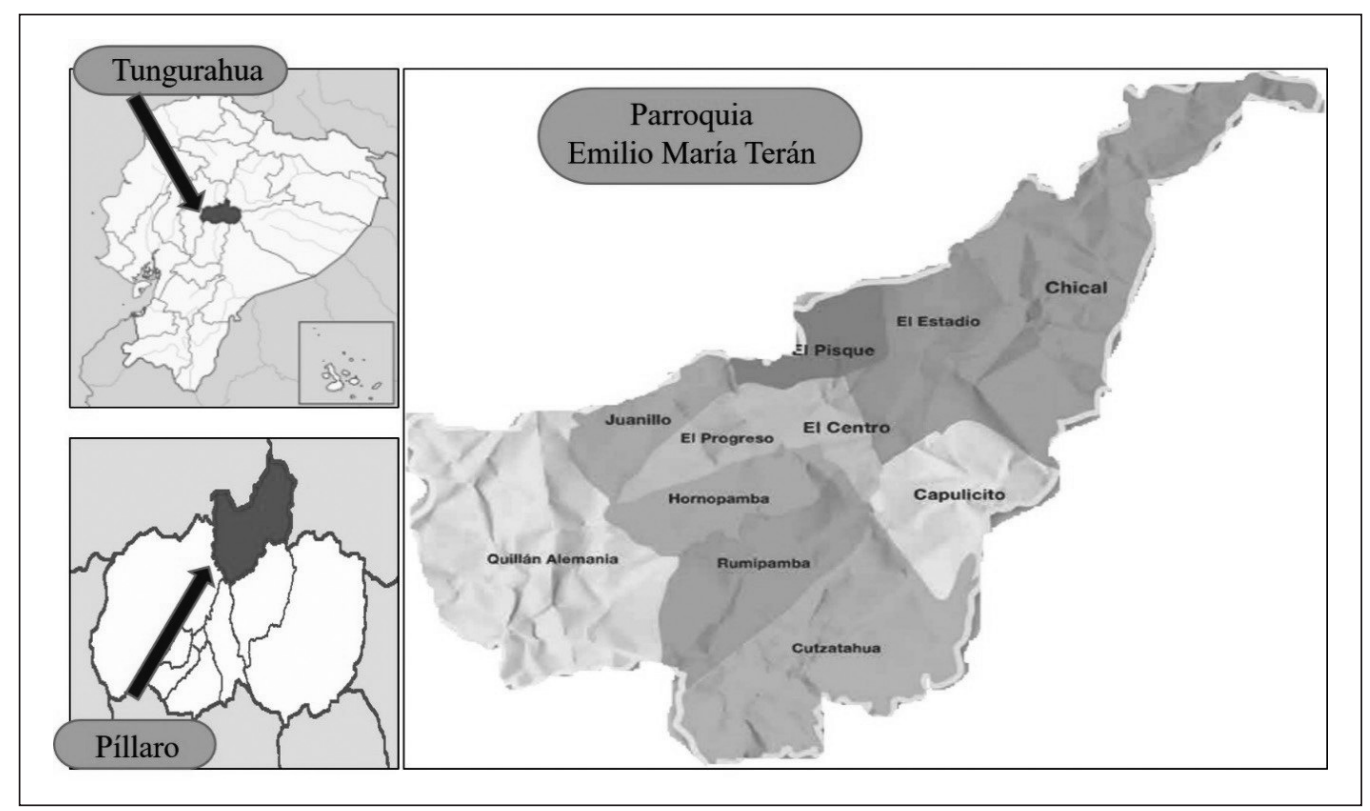

Figura 1. Ubicación del sitio donde se realizó la investigación

\subsection{Diseño de estudio}

Este trabajo está basado en información empírica. Los datos se analizaron por medio del software de análisis estadístico IBM SPSS Statistics Base, es decir, para las variables nominales o categóricas se aplicó la frecuencia con gráficos de barras, para las variables continuas se aplicó procedimientos descriptivos, se consultó y se analizó información en el Plan de Ordenamiento Territorial de la parroquia Emilio María Terán, para obtener datos como producción agrícola, distribución de la población por barrios. Por otra parte, el Gobierno Autónomo Descentralizado Parroquial de Emilio María Terán, cuenta con 25 análisis de suelos realizados por el INIAP, en diversos barrios, en el año 2015.

\subsection{Fuentes de información}

La presente investigación se basó en dos tipos de fuentes de información: bibliográfica y empírica, en esta última se compara el trabajo de campo con los resultados obtenidos mediante estudios científicos, los mismos que fueron realizados por algunas instituciones en torno a la temática sobre degradación de suelos. 1. Información primaria: 196 encuestas cerradas, 25 análisis de suelos para identificar el promedio de macro y micronutrientes presentes en los suelos de la parroquia. 2. Secundaria: revisión bibliográfica de documentos de la Organización de las Naciones Unidas para la Alimentación y la Agricultura (FAO), Programa Mundial de Alimentos, Facultad Latinoamericana de Ciencias Sociales (FLACSO), Instituto Nacional de Estadística y Censos de Ecuador (INEC), Gobierno Autónomo Descentralizado de la provincia de Tungurahua - Plan de Desarrollo y Ordenamiento Territorial de la parroquia Emilio María Terán 2015-2019 (PDOT EMT) y el Ministerio de Agricultura y Ganadería del Ecuador (MAG).

\subsection{Población y muestra}

El universo de este estudio se conformó con la población de la parroquia Emilio María Terán, perteneciente al cantón Santiago de Píllaro, la misma que cuenta con 397 familias que se encuentran dedicadas a diferentes actividades agropecuarias como principal fuente de ingreso (GAD de Emilio María Terán, 2015).

Para realizar el cálculo del tamaño de la muestra conociendo la población total se aplicó la ecuación [1], con un nivel de confianza del $95 \%$, un margen de error del $5 \%$ y una población de 397. 


$$
n=\frac{N \times z^{2} \times p \times q}{d^{2} \times(N-1)+z^{2} \times p \times q} ; n=\frac{397(1,96)^{2} \times 0,5 \times 0,5}{(0,05)^{2} \times(397-1)+(1,96)^{2} \times 0,05 \times 0,05}
$$

Donde:

- $\mathrm{N}=$ población total

- $\quad \mathrm{Z}=$ nivel de confianza,

- $\quad \mathrm{P}=$ proporción de individuos con características deseadas $(0,5)$

- $\quad \mathrm{Q}=$ probabilidad de fracaso

- $\quad \mathrm{D}=$ nivel de precisión (Error máximo admisible en términos de proporción).

- $\mathrm{n}=$ muestra

La muestra de esta investigación se compuso de 196 hogares agrícolas a quienes se aplicó una encuesta.

\subsection{Recolección de información}

En la presente investigación se utilizó como herramienta para recolectar la información una encuesta cerrada estructurada con 51 preguntas distribuidas en tres secciones: a) datos socioeconómicos (edad, género, estado civil, nivel de educación y miembros del hogar), b) datos de producción agropecuaria (área, producción actual de la UPA, disminución de la productividad, tipo de riego, tipo de insumos que utiliza y cultivos que produce en su UPA), y c) degradación del suelo (color, topografía, calidad del suelo, tipo de labranza, presencia de cangahuas y tipo de erosión), para determinar su efecto sobre el ingreso económico de los hogares agrícolas de la parroquia.

\subsection{Análisis inferencial}

Para probar la hipótesis central "si existe relación entre degradación del suelo y la situación socioeconómica de los agricultores en la agricultura familiar de la parroquia Emilio María Terán", se realizó un análisis de regresión logística binaria entre las variables predictoras $(\mathrm{X})$, consideradas como determinantes de la degradación del suelo, con la variable dependiente o de criterio (Y), la cual tiene una codificación de 1 si no posee áreas degradadas, y 0 si tiene áreas degradadas que no cultiva.

Se efectuó un análisis de regresión logística binario, según las características presentadas por la variable dependiente, con la ayuda del modelo logit, la regresión pretende determinar la probabilidad de que el proceso de degradación del suelo aumente o disminuya según las variables predictoras en estudio. La ecuación de estudio del modelo de regresión logística binaria se detalla en la ecuación [2].

$$
\text { logit }=\left(\pi_{i}\right)=\log \frac{\pi_{i}}{1-\pi_{i}}=\beta_{0}+\beta_{1} X_{1 i}+\ldots \beta_{j} X_{j i}
$$

La ecuación teórica para el modelo se presenta en la ecuación [3].

$$
\begin{array}{r}
\operatorname{logit}\left(p_{i}\right)=\ln \left(p_{i} / 1-p_{i}\right)=\beta_{0}+\beta_{1}\left(A U P A_{h a}\right)+\beta_{2}(I E M A E P)+\beta_{3}\left(S A C_{5}\right)+ \\
\beta_{4}(I E C S B U)+\beta_{5}(A E R)+\beta_{6}(U P A B)+\beta_{7}(D P U P A)+\beta_{8}(T L S)+\beta_{9}(M S U P A)+ \\
\beta_{10}(D M O)+\beta_{11}(P C)
\end{array}
$$

Donde:

- AUPAha: área de la UPA en ha

- IEMAEP: ingresos económicos mensuales actividad económica primaria.

- SAC5: situación actual en comparación hace 5 años.
- IECSBU: ingresos económicos en comparación al salario básico unificado USD 394.

- AER: actividad económica es rentable.

- UPABB: unidad de producción agrícola con áreas de bosque o barbecho. 
- DPUPA: disminución de productividad de su UPA en los últimos 5 años.

- TLS: tipo de labranza que realiza al suelo.

- MSUPA: manejo que le da al suelo de su unidad de producción agropecuaria.

- DMO: disminución de materia orgánica.

- PC: presencia de cangahuas.

\subsection{Variables de estudio}

Las variables de estudio que se analizaron en esta investigación corresponden a la información socioeconómica: edad, género, estado civil, miembros del hogar agrícola, actividad a la que se dedican, y su ingreso económico, como se observa en la Tabla 1.

Tabla1. Variables de datos socioeconómicos

\begin{tabular}{lc}
\hline \multicolumn{1}{c}{ Variable } & Indicador \\
\hline Edad & Educación \\
Años & Masculino/Femenino \\
Género & Nivel de educación \\
Estado civil & Soltero/casado/viudo \\
Miembros del hogar & Número de personas \\
UPA en la que vive & Propia/arrendada/prestada \\
Actividad que realiza & Agricultura/ganadería \\
Ingreso económico & Semanal/mensual/anual \\
Otra actividad & Jornalero/chofer/otro \\
\hline
\end{tabular}

Las variables de datos de producción agropecuaria que se presentan en la Tabla 2, se refieren al tamaño /área de la UPA, tipo de cultivo existente, total de producción; y ayudaron a tener un enfoque más concreto en la determinación de productividad y rentabilidad de las UPA.

Tabla 2. Variables de datos de producción agropecuaria

\begin{tabular}{lc}
\hline Variable & Indicador \\
\hline Superficie UPA & $\mathrm{ha}$ \\
Producción UPA & $\mathrm{kg} / \mathrm{ha}$ \\
Cultivos & Anuales y perennes \\
Disminución de la Productividad & $1 / 0$ \\
\hline
\end{tabular}

Las variables que se emplearon para determinar el nivel de degradación del suelo de la parroquia Emilio María Terán, son: color del suelo, topografía, área degradada, tipo de erosión, suelo degradado, eventos climatológicos, tipo de labranza, tipo de riego, como se observa en la Tabla 3.

Tabla 3. Variables de datos de degradación del suelo

\begin{tabular}{lc}
\hline \multicolumn{1}{c}{ Variable } & Indicador \\
\hline Color del suelo & Negro/rojo/gris \\
Topografía & Plano/quebrada/ladera \\
Área degradada & ha \\
Tipo de erosión & Eólica/hídrica/química/antropogénica \\
Suelo degradado & $1 / 0$ \\
Eventos climatológicos & Sequías/heladas/plagas \\
Temporalidad & Anual/semestral/trimestral/mensual \\
Tipo de labranza & Animal/manual/tractor/siembra directa \\
\hline
\end{tabular}




\subsection{Análisis de suelo y calicata}

Con la información proporcionada por los 25 análisis de suelo elaborados en los diferentes barrios de la parroquia Emilio María Terán, se realizó un cálculo de todos los macronutrientes, como son el nitrógeno, fósforo y potasio (N-P-K), y los micronutrientes: azufre $(\mathrm{S})$, calcio $(\mathrm{Ca})$, manganeso $(\mathrm{Mn})$, magnesio $(\mathrm{Mg})$, boro $(\mathrm{B})$, aluminio $(\mathrm{Al})$, sodio $(\mathrm{Na})$, de los cuales se obtuvo un promedio por cada elemento y se compararon estos resultados con los rangos establecidos para cada macro y micronutriente, llegándose a verificar el rango establecido para cada uno, es decir, si es alto, medio o bajo.

Se realizó una calicata en el sitio de estudio, donde se observó directamente los diferentes tipos de horizontes; el principal indicador es el color del suelo, posteriormente se realizó la medición de los horizontes.

\section{Resultados y discusión}

\subsection{Características socio económicas de la población}

La parroquia Emilio María Terán, está conformada mayoritariamente por el género femenino en un $52 \%$, mientras que el masculino representa el 48 $\%$, hecho que determina que la jefatura de los hogares agrícolas en esta parroquia esté en manos de las mujeres, estas cifras corroboran los datos publicados en el Plan de Ordenamiento Territorial de la parroquia, así como en el censo poblacional del INEC (2010) donde se estableció que en la parroquia viven 733 hombres (48,67\%), y 773 mujeres $(51,32 \%)$. En la Tabla 4 se observa que la edad promedio de la población en esta parroquia es de 51 años, los pobladores poseen un nivel de educación secundaria, el número promedio de miembros del hogar se ubica en cuatro personas. En su estudio Alvarez Gila et al.
(2008), basados en información proporcionada por el INEC, afirman, en lo referente al nivel de instrucción de los pobladores, que el $8 \%$ de los hombres de Ecuador son iletrados, y de igual manera el $12 \%$ de las mujeres. La cifra correspondiente al número promedio de miembros del hogar en la parroquia de estudio corrobora los datos revelados en la Encuesta Nacional de Ingresos y Gastos (ENIGHUR 20112012), la cual estableció que en la superficie urbana el promedio de personas que conforman un hogar es de 3,8 personas, mientras que en la zona rural las familias las integran 4 personas.

Al solicitar a los encuestados evaluar su nivel de ingresos económicos resultantes de la actividad agrícola, y compararlo con el salario básico unificado establecido en el Ecuador (USD 394), el $41 \%$ de los pobladores indicó que su nivel de ingresos es regular, el $15 \%$ afirmó que su nivel de ingresos es pobre; estas cifras corroboran la aseveración de Alvarez Gila et al. (2008), quienes afirman que en Ecuador el $20 \%$ de la población posee apenas el $2,5 \%$ de los ingresos económicos, mientras que el $20 \%$ de los ricos obtiene el 58,5\% de los ingresos. En la parroquia Emilio María Terán los jefes de hogar manifiestan que sus ingresos económicos al compararlos con la canasta básica son, en el $21 \%$ de los casos aceptables y buenos, y en el $2 \%$ de los casos los consideran muy buenos. En este estudio se determinó que el ingreso económico que se genera con la agricultura, en un $79 \%$ corresponde a USD 311,53 en comparación con el salario básico unificado (SBU), que en Ecuador está establecido en USD 394; si la actividad agrícola genera estos ingresos económicos no es el del todo rentable ya que la canasta familiar básica (CFB) se ubica en USD 719,88 (Figura 2). Estos datos corroboran las cifras presentadas por Álvarez Gila et al. (2008), quienes afirman que las tasas de pobreza urbana corresponden al 69 $\%$ y en el área rural la pobreza se ubica en el $80 \%$.

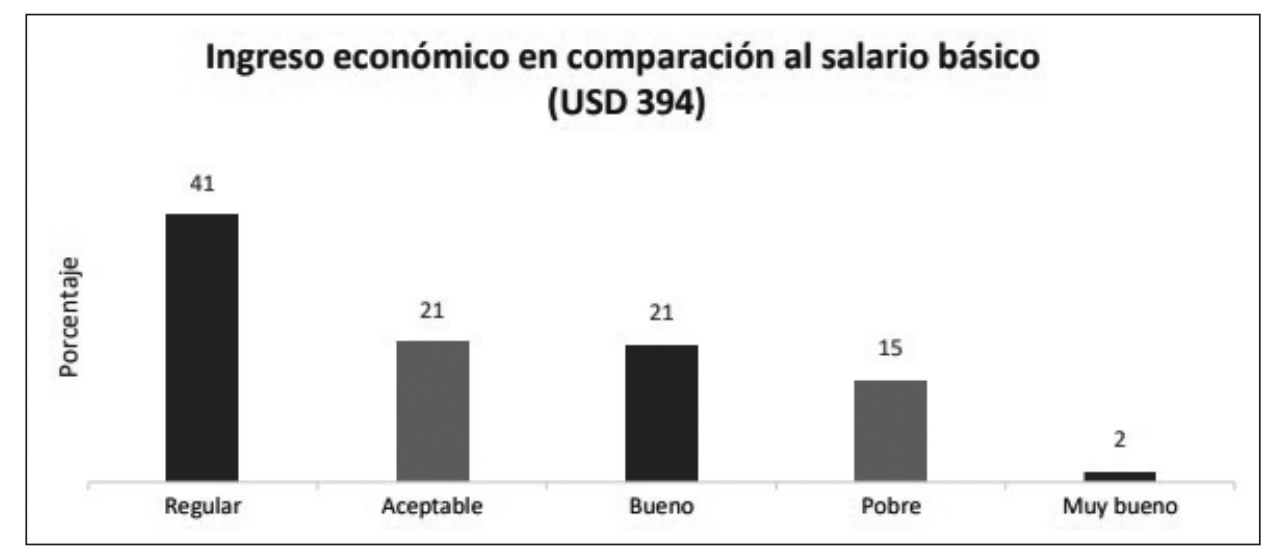

Figura 2. Ingreso económico en comparación al salario mínimo 
Tabla 4. Características socioeconómicas de la población

\begin{tabular}{lcc}
\hline & Características & Píllaro N=196 \\
\hline \multirow{2}{*}{ Género (\%) } & Femenino & 52 \\
& Masculino & 48 \\
\hline \multirow{2}{*}{ Nivel de educación (\%) } & Primaria & 42,3 \\
& Secundaria & 49 \\
& Técnica & 7,1 \\
& Universitaria & 1,5 \\
\hline & Soltero & 5,6 \\
Estado civil (\%) & Casado & 75 \\
& Unión libre & 9,7 \\
& Divorciado & 2,6 \\
& Viudo & 6,1 \\
\hline Edad promedio (años) & Separado & 1,0 \\
\hline \multirow{2}{*}{${ }^{\circ}$ de miembros del hogar(\# personas) } & & 51,79 \\
\hline
\end{tabular}

Los resultados de la encuesta de esta investigación colocan a la agricultura como la principal actividad económica en la parroquia Emilio María Terán, con el $74 \%$, seguido de la ganadería con el $19,4 \%$, el 5,6 \% corresponde a la floricultura y solo el $1 \%$ se dedica a otra actividad como se observa en la Tabla 5. Los datos del III Censo Nacional Agropecuario a nivel provincial señalan que en Tungurahua la actividad agropecuaria corresponde al $62,10 \%$ y el $37,89 \%$ a otras actividades no agropecuarias.

En lo referente a la tenencia de la tierra, los encuestados señalan que el $92,3 \%$ de las UPA es propia, el 5,1\% es rentada, y el 2,6\% es prestada; estas cifras revelan que la situación de la tenencia de la tierra ha mejorado, debido a que según los autores
Jordán Bucheli \& Sánchez Mena (2010), y sobre la base de un censo agropecuario realizado en el año 2000 , en el $68,4 \%$ de las UPA, los propietarios tenían títulos sobre las mismas, esta cifra correspondía al 71,9 \% del área agrícola total del país; y, por el contrario, el 6,7\% de las UPA eran trabajadas sin un título de propiedad. En este sentido, Gray (2009) afirma que la mayoría de los pobres del sector rural acceden a tierras para cultivo a través de la renta o de préstamo; la tenencia de la tierra es el primer medio de trabajo en el área rural de Ecuador y la carencia de este recurso es el principal motivo para la migración de sus habitantes. Por el contrario, Jordán Bucheli \& Sánchez Mena (2010) afirman que solo el $6,7 \%$ de los pobladores del sector rural que no posee tierra, migraría.

Tabla 5. Actividad económica primaria

\begin{tabular}{lcc}
\hline \multirow{2}{*}{ Características } & & $\begin{array}{c}\text { Píllaro } \\
\text { N=196 }\end{array}$ \\
\hline \multirow{3}{*}{ UPA (\%) } & Propia & 92,3 \\
& Rentada & 5,1 \\
& Prestada & 2,6 \\
\hline \multirow{2}{*}{ Actividad económica primaria (\%) } & Agricultura & 74 \\
& Ganadería & 19,4 \\
& Fruticultura & 5,6 \\
& Otro & 1 \\
\hline \multirow{2}{*}{ Actividad económica es rentable (\%) } & No & 69,4 \\
& Sí & 30,6 \\
\hline
\end{tabular}


E1 $82 \%$ de la población de la parroquia Emilio María Terán señala que no posee otra actividad económica secundaria, el 5,6\% indica que realiza actividades de jornalero, el 5,1\% posee un negocio propio, mientras que el resto se dedica a actividades como empleado público, empleado privado, albañil y otros en un bajo porcentaje. Al comparar estas cifras con aquellas relativas al Plan de Ordenamiento Territorial (GAD de Emilio María Terán, 2015), se constata que la parroquia Emilio María Terán se caracteriza por ser netamente agrícola-ganadera (Figura 3).

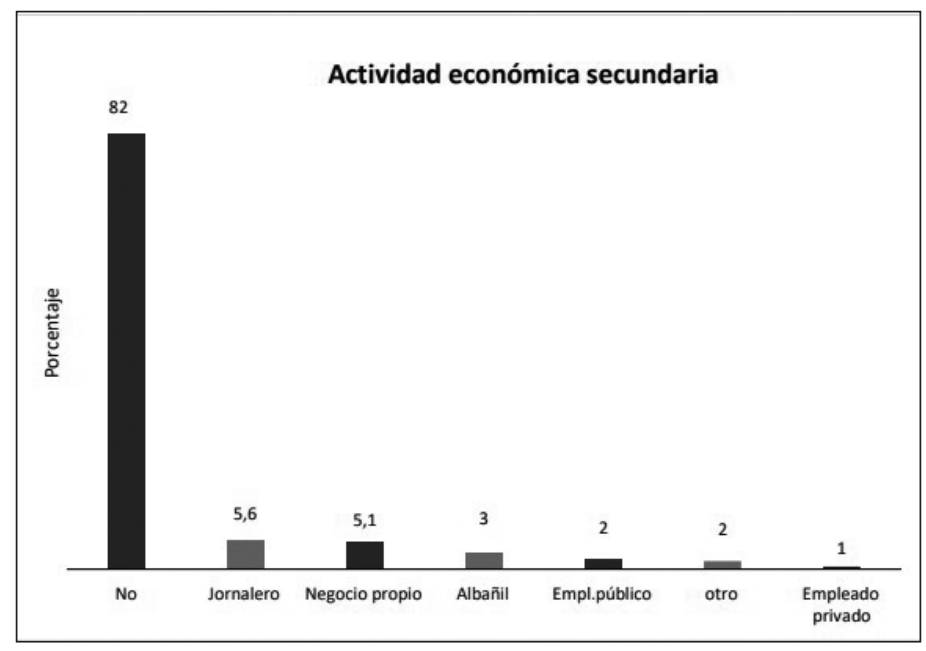

Figura 3. Actividad económica secundaria

\subsection{Principales características de la producción agrícola}

En la parroquia Emilio María Terán los encuestados manifiestan que la producción de la UPA es regular en el 33,7 \%, buena en el 33,2 \%, aceptable en el $27,0 \%$, muy buena $3,6 \%$ y pobre el $2,5 \%$. La principal actividad económica que realizan es la agropecuaria, los principales cultivos agrícolas de la UPA son: maíz (Zea mays), papa (Solanum tuberosum), fréjol (Phaseolus vulgaris), trigo (Triticum aestivum), haba
(Vicia faba), tomate de árbol (Solanum licopersicum), aguacate (Persea americana Miller), mora (Rubus glaucus), babaco (Carica pentagona), como se observa en la Figura 4. De Noni \& Trujillo (1986) en su estudio Degradacion del suelo en el Ecuador, afirman que en la antigüedad los agricultores aprovechaban la diversidad de microclimas de la Sierra, diversificando sus cultivos en función de cada piso ecológico; según el ESPAC (INEC, 2012), en la provincia de Tungurahua el $27,1 \%$ de los cultivos corresponde a tomate de árbol, el 8,2\% corresponde a la producción de papa.

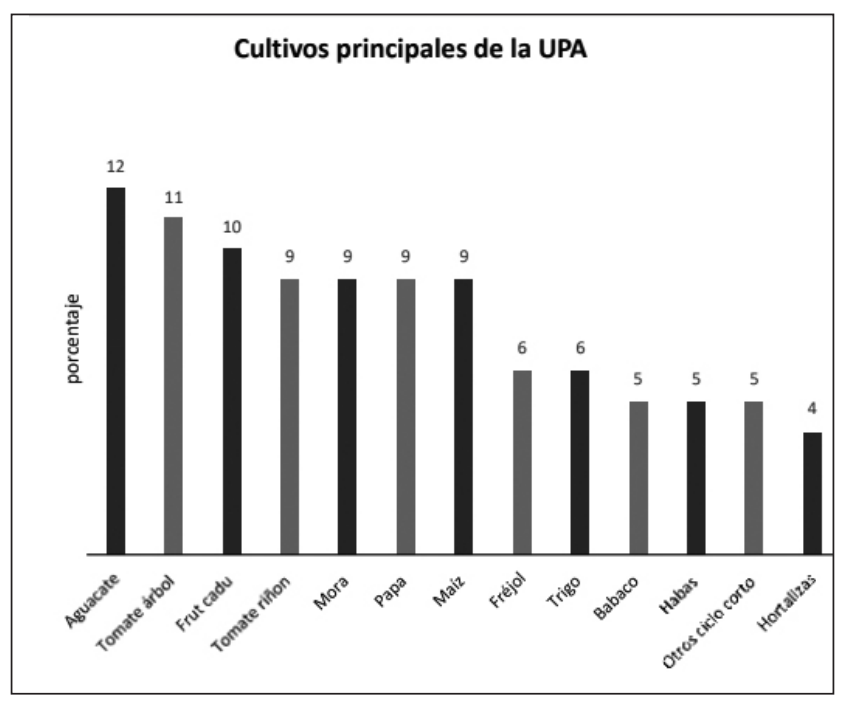

Figura 4. Cultivos principales de la UPA en \% 
En su estudio, Gray \& Bilsborrow (2018) concluyen que en el sector rural ecuatoriano es común que los productores complementen los bajos ingresos originados por la actividad agrícola, con aquellos generados por la actividad pecuaria. Este hecho se cumple entre los pobladores de la parroquia Emilio María Terán, quienes tienen como segunda actividad económica a la pecuaria, que les permite generar otro ingreso monetario; dentro de este rubro se encuentran: la crianza de cuyes con el $55 \%$, seguido de la crianza de aves de corral que corresponde al $22 \%$, el $15 \%$ se dedica a la cunicultura, y menos del $5 \%$ se dedican al manejo de ganado bovino, el $2 \%$ de porcino y tan solo el $1 \%$ corresponde a equinos, como se observa en la Figura 5. Si se comparan las cifras recabadas en este estudio con aquellos datos del Plan de ordenamiento territorial de Emilio María Terán (GAD de Emilio María Terán, 2015), en la parte pecuaria la crianza de cuyes concentra el $75 \%$, seguido de aves de corral con el $17 \%$, también el $4 \%$ pertenece a bovinos, y porcinos el $4 \%$, lo que indica que es una actividad económica importante ya que los productores basan su alimentación y economía en este sector, especialmente por la venta de subproductos de forma diaria o quincenal.

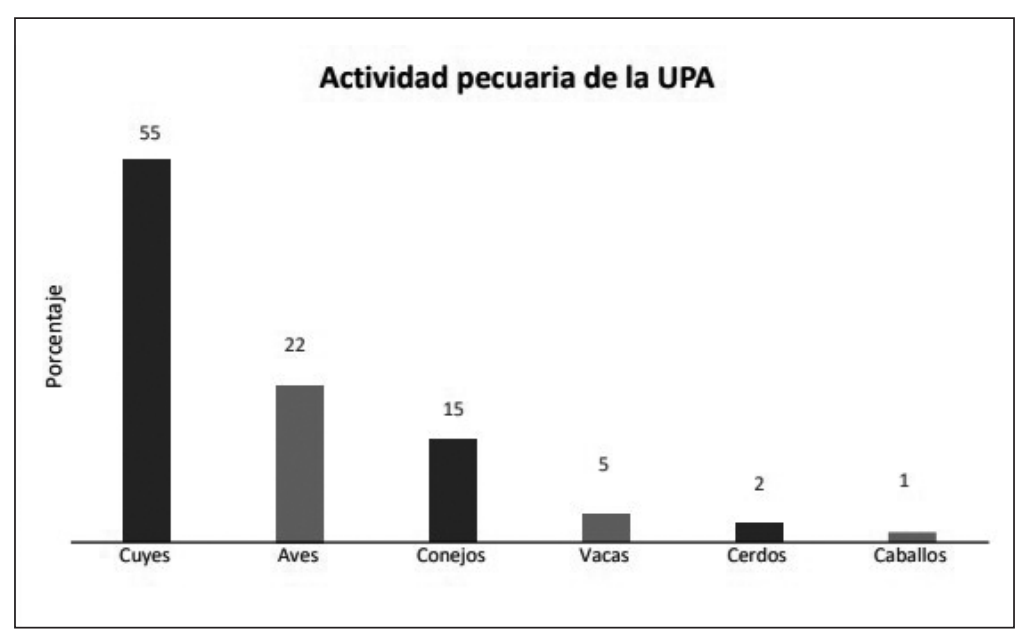

Figura 5. Actividad pecuaria

\subsection{Características cualitativas del suelo}

Debido a que la actividad agrícola es considerada como la actividad productiva que mayoritariamente acelera los procesos de erosión de suelos (Harden, 2001), en este estudio se analizaron las características de los suelos y las técnicas de labranza comúnmente empleadas por los pobladores de la parroquia Emilio María Terán. Se determinó que el $38,8 \%$ de los pobladores emplean labranza mixta (tractor y manual), el 34,2\% utilizan únicamente tractor, el 20,4\% lo hace de forma manual, y el 6,6 $\%$ utiliza tracción animal. Los autores Winters et al. (1998) afirman que los pequeños agricultores son los que primero valoran y adoptan nuevas tecnologías en el agro; en su estudio determinaron que el 68,2 \% de los agricultores han adoptado nuevas tecnologías. De Noni \& Trujillo (1986) afirman que el hombre y las actividades agrícolas que realiza en la tierra son los responsables en mayor medida de la degradación de los suelos. Álvarez Gila et al. (2008) coinciden en señalar que son las actividades agrícolas las que co- adyuvan a la erosión de los suelos, así como el grado de pendiente de los terrenos. Los terrenos ubicados en laderas son más propensos a la erosión (Winters et al., 1998). En este estudio se determinó que el $42,9 \%$ de los encuestados define la topografía de su UPA como plana, el 35,7 \% de topografía ondulada, un $18,4 \%$ posee topografía de ladera y solo el $3,1 \%$ pertenece a una quebrada (Tabla 6). Gray (2009) en su estudio Environment, land and rural out-migration in the southern Ecuadorian Andes, afirma que la tenencia de un terreno plano facilita el acceso y movilidad de las personas, convirtiendo a esta condición geográfica en un capital preciado. Contrariamente a lo aseverado por los encuestados en este estudio, en el Plan de Ordenamiento Territorial de la parroquia Emilio María Terán (GAD de Emilio María Terán, 2015), se señala que el $40,39 \%$ de los terrenos de la zona son relieves montañosos, con colinas altas el $20 \%$, vertientes cóncavas y convexas el 39,14 \%; todos estos antecedentes permiten determinar que la topografia en este territorio es irregular. En cuanto al color del suelo de la parroquia, es negro $83,7 \%$, 
con altos contenidos de materia orgánica del suelo (MOS), aunque los análisis químicos indican que posee un promedio de 3,0-5,0\% de MOS, ubicándolo como un contenido medio; el suelo color gris alcanza el 12,2 \% (Tabla 6).

En este estudio se determinó que el 86,2\% de los productores agrícolas afirman que en su UPA se presenta disminución de la materia orgánica en el suelo, esta cifra corrobora las aseveraciones de Eche Enríquez (2013) en su estudio Land degradation, small-scale farms' development, and migratory flows; the case of Tapachula/Chiapas, quien afirma que la degradación de los suelos en las regiones analizadas, se constituye en un obstáculo para el desarrollo, reduciendo el tamaño del área productiva de la UPA, la fertilidad del suelo y la productividad de los cultivos.

Tabla 6. Datos de la degradación del suelo

\begin{tabular}{lcc}
\hline \multirow{2}{*}{ Características } & & Píllaro \\
& & N=196 \\
\hline & Negro & 83,7 \\
\multirow{2}{*}{ Color del suelo UPA (\%) } & Rojo & 1 \\
& Amarillo & 2,6 \\
& Gris & 12,2 \\
& Otro & 0,5 \\
\hline \multirow{2}{*}{ Topografía UPA (\%) } & Plana & 42,9 \\
& Ondulada & 35,7 \\
& Quebrada & 3,1 \\
& Ladera & 18,4 \\
\hline \multirow{2}{*}{ Tipo de labranza que realiza } & Animal & 6,6 \\
& Manual & 20,4 \\
& Tractor & 34,2 \\
\multirow{2}{*}{ Disminución de M.O. en el suelo } & Mixtas & 38,8 \\
\hline \multirow{2}{*}{ Presencia de cangahuas } & No & 13,8 \\
& Si & 86,2 \\
\hline
\end{tabular}

En la Tabla 7 se observa que la principal fuente de contaminación del suelo es el uso de agroquímicos, con el $82,9 \%$, mientras que las aguas negras y basura de los poblados corresponden al 16,5 \%, y el 0,6 \% a los desechos industriales. De los diferentes tipos de erosión que afectan al suelo de la parroquia, en primer lugar, se encuentra la erosión química con el $50 \%$, la erosión hídrica con el 47,4\%, y por último con el $2,6 \%$ la erosión eólica. En lo que se refiere a los diferentes efectos climatológicos que también afectan a la UPA se encuentra las sequías con el $74 \%$, seguido de las heladas con el 12,2\%, el 7,7 \% corresponde a plagas, el 2,0 \% a inundaciones, el 3,6 \% a erosión, y el $0,5 \%$ a tala de bosques. Gray \& Bilsborrow (2018) en su estudio Environmental Influences on Human Migration in Rural Ecuador afirman que los factores ambientales que más inciden en la degradación de los suelos son la topografía de la UPA, el acceso al agua de regadío y la variación de las precipitaciones en la zona. El Plan de ordenamiento territorial de Emilio María Terán (GAD de Emilio María Terán, 2015), revela que el 28,98\% del territorio está expuesto a una severa erosión, el 26,20\% de la superficie parroquial presenta alta incidencia de erosión, el 32,6 \% del territorio registra una moderada erosión, y finalmente el $12,22 \%$ presenta baja tendencia a la erosión.

Tabla 7. Efectos climatológicos que afectan al suelo

\begin{tabular}{ccc}
\hline & Características & Píllaro \\
& & $\mathbf{N}=\mathbf{1 9 6}$ \\
\hline \multirow{2}{*}{ Fuentes de contaminación (\%) } & Uso de agroquímicos & 82,9 \\
& Aguas negras y basura de los poblados & 16,5 \\
& Industria & 0,6 \\
\hline Eventos climatológicos que afecta a su UPA (\%) & Sequias & 74 \\
& Inundaciones & 2 \\
& Heladas & 12,2 \\
& Plagas & 7,7 \\
& Erosión-degradación-salinización -suelos & 3,6 \\
\hline
\end{tabular}




\begin{tabular}{ccc}
\hline & Anual & 66 \\
& Semestral & 17 \\
Frecuencia que ocurre el evento (\%) & Trimestral & 6,2 \\
& Mensual & 7,2 \\
& Quincenal & 2,1 \\
& Semanal & 1,5 \\
\hline \multirow{2}{*}{ Tipo de erosión que afecta la UPA (\%) } & Hídrica & 47,4 \\
& Eólica & 2,6 \\
\hline
\end{tabular}

La degradación del suelo en la parroquia Emilio María Terán ocasiona efectos negativos como se observa en la Tabla 8, principalmente la disminución de la productividad en un 96,3\%, contaminación del suelo de la UPA con el 80,6\%. Estas cifras confirman las aseveraciones de Alvarez Gila et al. (2008), quienes manifestaron en su estudio que en la Sierra centro de Ecuador, y como consecuencia de la degradación de los suelos, la productividad de las unidades agrícolas ha disminuido, así como los ingresos de los productores. De manera generalizada, Neumann et al. (2015) afirman que la degradación de los suelos provoca pérdida de productividad; similar criterio comparten los autores Bolt et al. (2005) en su estudio Estimating the cost of environmental degradation, al evaluar los cambios en la producción agrícola, determinaron que la degradación de los suelos provoca un declive en la cantidad de cosecha que se recoge.

Gray \& Bilsborrow (2018) afirman que factores ambientales, como el acceso al agua de regadío, calidad de la tierra, promedio anual de precipitaciones, y la estacionalidad de las precipitaciones en la zona, están directa y positivamente relacionados con los ingresos económicos que genera la actividad agrícola. Por el contrario, Winters et al. (1998) manifiestan que existen solamente dos factores que inciden en la baja productividad de la actividad agrícola: la sobreexplotación (sobrepastoreo, rotación muy corta, excesivo uso de fertilizantes químicos) y la subinversión (falta de mantenimiento establecido). A manera de colofón, en su estudio Eche Enríquez (2013) afirma que los ingresos económicos de las familias de la agricultura rural se reducen debido a los procesos de degradación de los suelos.

Según el Plan de ordenamiento territorial de Emilio María Terán (GAD de Emilio María Terán, 2015) la migración de la población rural se produce de manera permanente cuando los destinos son cercanos a ciudades como Ambato y Píllaro y los retornos son diarios, esto implica una circulación de capital en gastos. En su estudio, De Noni \& Trujillo (1986) determinaron que el $50 \%$ de la superficie de Ecuador posee algún nivel de degradación, de ésta el $15 \%$ se encuentra en el callejón interandino a 1.500 y 3.000 m s.n.m. A pesar de estas realidades, en este estudio se determinó que el $68,1 \%$ de los encuestados no piensan migrar aun con la degradación presente en los suelos; este dato contradice a la aseveración de Neumann et al. (2015), quienes manifestaron que entre los principales inductores de la migración del campo están los desastres naturales, degradación de suelo, la desertificación y cambios en el clima. Por su parte, Gray \& Bilsborrow (2018) corroboran la existencia de cinco factores ambientales que provocarían la migración: acceso al agua, calidad del suelo, la topografía, promedio de precipitaciones, y la estacionalidad de precipitaciones en la zona. No coincide con estas afirmaciones el autor Gray (2009), quien afirma que las principales causas de migración del sector rural se deben a la pobre tenencia de la tierra más que a los factores atmosféricos o condiciones climáticas adversas, y más bien aduce que la migración del campo obedece a la estrategia de los pobladores rurales para diversificar sus ingresos y mitigar los riesgos de la producción agrícola debido a la escasez de créditos a los que no pueden acceder. Eche Enríquez (2013) en su estudio afirma que la degradación de los suelos es uno de los factores importantes para la migración de los agricultores rurales. En este estudio se determinó que el 85,6 \% de los encuestados señalan que no aplican ningún método para recuperar los suelos degradados. En el Plan de Ordenamiento Territorial de la parroquia Emilio María Terán se afirma que el recurso suelo presenta gran impacto por la quema de pajonales y bosques andinos, la presencia de hatos ganaderos, y el avance de la frontera agrícola, que evidencian un alto nivel de afectación. 
Tabla 8. Consecuencias de la degradación del suelo

\begin{tabular}{|c|c|c|}
\hline Características & & $\begin{array}{c}\text { Píllaro } \\
\mathrm{N}=196\end{array}$ \\
\hline \multirow{2}{*}{ Disminución de productividad debido a la degradación del suelo } & No & 3,7 \\
\hline & $\mathrm{Si}$ & 96,3 \\
\hline \multirow{2}{*}{ Piensa abandonar o migrar por la degradación del suelo } & No & 68,1 \\
\hline & $\mathrm{Si}$ & 31,9 \\
\hline \multirow{2}{*}{ Aplica algún método para recuperar suelos degradados } & No & 85,6 \\
\hline & $\mathrm{Si}$ & 14,4 \\
\hline \multirow{2}{*}{ Existen problemas de contaminación del suelo de su UPA } & No & 19,4 \\
\hline & $\mathrm{Si}$ & 80,6 \\
\hline
\end{tabular}

En su estudio Environmental Influences on Human Migration in Rural Ecuador, los autores Gray \& Bilsborrow (2018) determinan que para las mujeres la calidad de la tierra de la UPA es muy importante, así como para los hombres lo es la topografía de la tierra; en cambio la tasa media de precipitaciones en la zona, así como el acceso al agua de regadío, son factores muy importantes para los pobladores rurales más pobres. Con respecto a la topografía de la UPA de la parroquia en estudio, el mayor porcentaje corresponde a la topografía plana con el 42,9\%, lo que la hace menos propensa a la degradación. En la Tabla 9 se observa los datos obtenidos, donde se refleja que cerca del 50 $\%$ de la topografía del suelo es plana y recomendable para la agricultura y ganadería, ya que técnicamente no existe mayor tipo de erosión que afecte al suelo y se puede incrementar su productividad, al existir terrenos con topografía de tipo quebrada como los que se observan en la parroquia Emilio María Terán, se determina que estos no son muy aptos para la actividad agrícola y, por ende, los costos de producción aumentan y no se obtendrá una productividad adecuada, el problema en este tipo de topografía que compromete la actividad agrícola y ganadera, es la pendiente de la tierra: a mayor ángulo se evidencia mayor desgaste del suelo, y mayor rapidez de escorrentía y, por lo tanto, el agua baja a mayor velocidad acelerando la erosión (GAD de Emilio María Terán, 2015). Con respecto a la calidad del suelo de la parroquia analizada se puede señalar que el 33,2 \% de ésta posee buena calidad y tan solo el 1,5\% de tierra se identifica con una calidad de suelo considerada pobre.

Tabla 9. Calidad de suelo y topografía de la UPA

\begin{tabular}{ccc}
\hline Características & & $\begin{array}{c}\text { Píllaro } \\
\mathbf{N}=\mathbf{1 9 6}\end{array}$ \\
\hline & Plana & 42,9 \\
Topografía UPA (\%) & Ondulada & 35,7 \\
& Quebrada & 3,1 \\
& Ladera & 18,4 \\
\hline Calidad del suelo (\%) & Muy buena & 7,1 \\
& Buena & 33,2 \\
& Aceptable & 29,1 \\
& Regular & 29,1 \\
& Pobre & 1,5 \\
\hline
\end{tabular}

\subsection{Análisis logístico de regresión binaria}

Se correlacionaron las siguientes variables independientes (predictoras) recolectadas mediante la encuesta: área de la UPA, ingreso mensual, cómo consi- dera sus ingresos económicos en comparación al salario básico unificado de USD 394, cree que su actividad económica es rentable, tiene en su UPA áreas con bosque o barbecho, ha disminuido la productividad de su UPA en los últimos 5 años, qué tipo de labranza realiza al suelo, 
cómo es el manejo que usted le da al suelo de la UPA, cree que existe disminución de la materia orgánica, existe en su propiedad presencia de cangahuas.

Las Tablas 10 y 11 presentan los resultados del análisis logístico de regresión binaria como se mencionó en el punto 3.6, se demuestra que el modelo es significativo $(\chi=30,770 ; p \leq 0,05)$; la bondad del ajuste del modelo es determinada por el test de Hosmer y Lemeshow como no significativo $(\chi=11.864 ; \mathrm{gl}=8 ; p \geq 0,05)$, lo cual indica que no existe evidencia de un ajuste pobre.

Tabla 10. Prueba de ómnibus del modelo de coeficientes

\begin{tabular}{lcccc}
\hline & Chi cuadrado & Gl & Sig. \\
\hline Paso & 30,770 & 11 & 0,001 \\
Paso 1 & Bloque & 30,770 & 11 & 0,001 \\
& Modelo & 30,770 & 11 & 0,001 \\
\hline
\end{tabular}

Tabla 11. Test de Hosmer y Lemeshow

\begin{tabular}{cccc}
\hline Paso & Chi cuadrado & Gl & Sig. \\
\hline 1 & 11,864 & 8 & 0,157 \\
\hline
\end{tabular}

Para un análisis de regresión logística, el bloque uno demuestra que existe el $73 \%$ de credibilidad de acierto en el resultado de la variable dependiente (VD), cuando se conoce si tiene áreas degradadas que no se cultivan.

En la Tabla 12 se presentan los estimadores de los parámetros (coeficientes B), grados de libertad, significancia y el exponente beta $(\operatorname{Exp}(B))$, para las variables predictoras y la constante del modelo, fueron incorporadas once variables predictoras: área de la UPA (AREA_UPA_LOG), cuánto genera mensualmente de la actividad económica primaria (INGRESO_ECON_LOG), situación actual en comparación con hace 5 años (Situa_actual), ingresos económicos en comparación al salario básico unificado USD 394 (Ingreso_econ_), actividad económica rentable (Activ_econ_rentable), unidad de producción agrícola con áreas de bosque o barbecho (UPAS_bosque_barbecho), disminución de la productividad de su UPA en los últimos 5 años (Dismin_product_UPA), tipo de labranza que realiza al suelo (Labranza suelo), manejo que le da al suelo de su unidad de producción agrícola (Manejo_suelo_UPA), disminución de la materia orgánica (Dismin_materia_organ), presencia de cangahuas (Presencia cangahua), las variables que no aparecen fueron desechadas porque sus coeficientes no difieren significativamente de 0 , es decir, que no aportan a la predicción de degradación del suelo.

Interpretando los valores del coeficiente $\beta$ se puede decir que la variable áreas de bosque o barbecho $(\beta=-1,156 ; p \leq 0,05)$, significativamente infiere negativamente sobre la degradación del suelo, indicando que las unidades de producción agrícola con áreas de barbecho y bosques tienen menos probabilidades de degradación. Al ser el $\operatorname{Exp}(\mathrm{B})$ menor a 1 se obtiene la inversa $(1 / 0,315=3,17)$. El incremento de una unidad de bosque o barbecho disminuye en 3,1 veces la probabilidad de presencia de áreas degradadas en la UPA. Adicionalmente, la variable ingresos económicos $(\beta=, 119 ; p \geq 0,05)$ obtenidos por el jefe de hogar no es significativo, ya que la magnitud del área degradada aun no infiere sobre la generación de ingresos. 
Tabla 12. Variables en la ecuación

\begin{tabular}{|c|c|c|c|c|c|}
\hline & Variables & B & gl & Sig. & $\operatorname{Exp}(B)$ \\
\hline \multirow{11}{*}{ Paso $1^{a}$} & AREA_UPA_LOG & 0,231 & 1 & 0,636 & 1,26 \\
\hline & INGRESO_ECON_LOG & 2,012 & 1 & 0,111 & 7,48 \\
\hline & Situa_actual & 0,058 & 1 & 0,82 & 1,06 \\
\hline & Activ_econ_rentable(1) & 0,518 & 1 & 0,297 & 1,678 \\
\hline & UPAS_bosque_barbecho(1) & $-1,156$ & 1 & $0,022 * *$ & 0,315 \\
\hline & Dismin_product_UPA(1) & $-0,422$ & 1 & 0,56 & 0,656 \\
\hline & Labranza_suelo & 0,016 & 1 & 0,941 & 1,016 \\
\hline & MANEJO_SUELO2 & $-0,06$ & 1 & 0,959 & 0,942 \\
\hline & Dismin_materia_organ(1) & $-20,547$ & 1 & 0,998 & 0 \\
\hline & Presencia_cangahua(1) & 0,306 & 1 & 0,587 & 1,358 \\
\hline & Constante & $-5,975$ & 1 & 0,068 & 0,003 \\
\hline
\end{tabular}

a Variable(s) introducida(s) en el paso 1: Situa_actual, Ingreso_econ_, Activ_econ_rentable, UPAS_bosque_barbecho,

Dismin_product_UPA, Labranza suelo, Manejo_suelo_UPA, Dismin_materia_organ, Presencia_cangahua

\section{Conclusiones}

La causa principal de la degradación del suelo en la parroquia Emilio María Terán es la provocada por la erosión antropogénica. Las causas secundarias de la degradación del suelo son el tipo de riego, el uso inadecuado de agroquímicos, y diferentes fuentes de contaminación.

El $79 \%$ de los pobladores de la parroquia Emilio María Terán posee un nivel de ingresos inferior al SBU, contribuyendo a la hipótesis de que los ingresos económicos de la agricultura familiar son inferiores al SBU. Según las encuestas realizadas, la principal actividad económica proviene de la agricultura, donde se destaca que la persona encargada de esta actividad es la femenina.

El contenido de los niveles de macronutrientes son rangos alto, medio, bajo; y de micronutrientes el

\section{Referencias}

Álvarez Gila, O., López de Maturana-Diéguez, V., \& Ugalde-Zaratiegui, A. (2008). Ecuador Case Study Report. EACH-FOR - Environmental Change and Forced Migration Scenarios.

Bolt, K., Ruta, G., \& Sarraf, M. (2005). Estimating the cost of environmental degradation: A tra- rango es alto, así como la materia orgánica se encuentra en rango medio.

Hay una relación directa entre los ingresos económicos y los factores sociales con la degradación del suelo por la baja productividad ya que existe un 70,9 $\%$ de las UPA con áreas degradadas que no se cultivan; como consecuencia de la degradación, la mayor parte de los pobladores reconocen que la producción de su UPA ha disminuido, y sí existe problemas de contaminación de suelo por las actividades antropogénicas.

Mediante el análisis de regresión se puede determinar la importancia de mantener el suelo en barbecho y con cobertura forestal, por su efecto en la reducción de las áreas degradadas. Además, cualitativamente se evidenció que existe una precaria situación económica y la presencia de UPAs con áreas degradadas, sin embargo, los análisis cuantitativos determinan que la degradación no tiene efecto sobre el ingreso económico.

ining manual in English, French and Arabic. Washington: World Bank.

De Noni, G., \& Trujillo, G. (1986). Degradación del suelo en el Ecuador. Cultura: Revista del Banco Central del Ecuador, 24a, 383-394. 
Eche Enríquez, D. (2013). Land degradation, small scale's farm development and migratory flows: the case of Tapachula/Chiapas. Kassel: Kassel University Press.

Espinoza Ramírez, M., Andrade Limas, E., Rivera Ortiz, P., \& Romero Díaz, A. (2011). Degradación de suelos por actividades antrópicas en el norte de Tamaulipas, México. Papeles de geografia, (53-54), 77-88.

GAD de Emilio María Terán. (2015). Plan de ordenamiento territorial Emilio María Terán 20152019. GAD de Emilio María Terán.

Gray, C. L. (2009). Environment, land, and rural out-migration in the southern Ecuadorian Andes. World Development, 37(2), 457-468.

Gray, C., \& Bilsborrow, R. (2013). Environmental influences on human migration in rural Ecuador. Demography, 50(4), 1217-1241. https:// doi.org/10.1007/s13524-012-0192-y

Harden, R. M. (2001). AMEE Guide No. 21: Curriculum mapping: a tool for transparent and authentic teaching and learning. Medical teacher, 23(2), 123-137. https://doi. org/10.1080/01421590120036547

INEC - Instituto Nacional de Estadísticas y Censos. (2012). Encuesta de Superficie y Producción Agropecuaria Continua ESPAC-2012. Quito: INEC.
Jiménez, S. (2011). La erosión del suelo en el Ecuador. En Estudio científico de los indices de erosión en el Ecuador. Observatorio de política ambiental.

Jordán Bucheli, F., \& Sánchez Mena, R. (2010). Land tenure characteristics and vulnerability to natural disasters in Ecuador. En Land tenure and natural disasters. Addressing land tenure in countries prone to natural disasters (pp. 99118). Roma: FAO.

Mateo Fernández, S.M. (2017). Evaluación de la degradación de suelos en dos agroecosistemas de la granja Santa Inés. Universidad Técnica de Machala, Unidad Académica de Ciencias Agropecuarias.

Morales, C. (2010). Degradación de las tierras en Ecuador. Quito: Mecanismo Mundial- CEPAL.

Neumann, K., Sietz, D., Hilderink, H., Janssen, P., Kok, M., \& van Dijk, H. (2015). Environmental drivers of human migration in drylands-A spatial picture. Applied Geography, 56, 116-126. https://doi.org/10.1016/j.apgeog.2014.11.021

Piscitelli, M. (2015). Degradación de suelos. Obtenido de: https://www.unicen.edu.ar/content/ degradaci\%c3\%b3n-de-suelos

Winters, P., Espinoza, P., \& Crissman, C. (1998). Manejo de los recursos en los Andes Ecuatorianos. Quito: Ediciones Abya-Yala. 